\title{
Endothelial Progenitor Cells and Vascular Repair
}

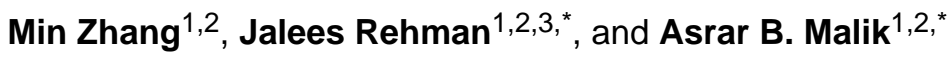 \\ ${ }^{1}$ Department of Pharmacology, University of Illinois, Chicago, IL \\ ${ }^{2}$ Center for Lung and Vascular Biology, University of Illinois, Chicago, IL \\ ${ }^{3}$ Department of Medicine, Section of Cardiology, University of Illinois, Chicago, IL
}

\begin{abstract}
Purpose of review-Identify recent advances in the field of vascular repair by regenerative endothelial cells (ECs) and endothelial progenitor cells (EPCs).

Recent findings-A growing number of studies indicate that bone marrow derived circulating EPCs do not engraft into blood vessels, but that such circulating cells may regulate vascular repair via paracrine mechanisms. Novel modes of paracrine regulation are being uncovered, such as the release of EC-derived microparticles or microvesicles which contain microRNAs that can promote vascular repair. Instead of circulating cells, tissue resident ECs or EPCs may primarily drive the restoration of vascular function after endothelial injury. In addition to the generation of ECs/EPCs from pluripotent stem cells, direct reprogramming of fibroblasts to ECs/EPCs is becoming an important source of regenerative vascular cells.
\end{abstract}

Summary-Ongoing efforts to understand the mechanisms that regulate vascular repair by resident regenerative vascular cells as well as their generation from fibroblasts and pluripotent stem cells will form the basis of future regenerative therapies.

\section{Keywords}

Endothelial progenitor cells; vascular repair; vascular regeneration; angiogenesis; vasculogenesis

\section{Introduction}

The concept that immature endothelial progenitor cells (EPCs) circulate in the adult blood and participate in postnatal angiogenesis or vascular repair was proposed by Asahara et al. in 1997[1]. This idea was met with great enthusiasm because it suggested that EPCs could be derived from peripheral blood and used as a novel therapy in patients with vascular disease, thus resulting in over 4,000 publications during the past 15 years. The original paper by Asahara et al[1] defined EPCs as mononuclear blood cells (MNCs) isolated from peripheral blood that were cultured in the presence of growth factors and were positive for the cell surface markers such as CD34 or FLK1. Numerous subsequent studies used variations of

\footnotetext{
*Please address correspondence to: Asrar B. Malik, Ph.D., abmalik@uic.edu or Jalees Rehman, M.D., jalees@ uic.edu, University of Illinois, College of Medicine, Department of Pharmacology, 835 S. Wolcott, Chicago, IL 60612, Phone: (312) 996-7636.

Conflicts of interest

The authors have no pertinent financial conflicts of interest.
} 
this approach and confirmed the endothelial phenotype of peripheral blood EPCs by demonstrating that these cells were able to take up acetylated LDL (acLDL) and express the endothelial surface adhesion molecule PECAM (CD31), and that they were indeed proangiogenic in a variety of ischemia models. The underlying hypothesis was that transplanted EPCs could regenerate the vasculature or promote blood vessel growth by providing proliferative endothelial cells. However, analysis of such EPCs derived from adult human peripheral blood revealed that the vast majority of cells previously deemed as "EPCs" were minimally proliferative cells derived from a myeloid-monocytic lineage [2]. This suggested that circulating monocytic "EPCs" did not promote vascular repair or angiogenesis by supplying new endothelial cells, but probably activated resident endothelial cells by releasing paracrine factors such as vascular endothelial growth factor (VEGF) or hepatocyte growth factor (HGF) [2].

The definition of what constitutes an EPC remains controversial, since monocytic-myeloid cells can transiently express cell surface markers typically found on endothelial cells and thus mimic an endothelial phenotype without necessarily meeting other criteria used that define a progenitor cell, such as the ability of a single progenitor cell to give rise to a proliferative colony of cells. Many studies have attempted to identify cell surface markers that are unique to EPCs and distinguish them from mature adult endothelial cells as well as from myeloid-monocytic cells, but these attempts have met little success. In the effort to solve this problem, Yoder et al [3] proposed to redefine EPCs by clonal analysis instead of cell surface antigen expression. They identified a very rare population of highly proliferative colony forming endothelial cells in preparations of cultured peripheral blood mononuclear cells (less than $0.0001 \%$ of the MNCs), which were indeed able to give rise to clonal colonies after 14 days culture and referred to this population as "endothelial colony-forming cells" (ECFCs). Even though such ECFCs behave like true progenitor cells and do not express myeloid or monocytic markers, it is unclear whether they play any significant role in physiological vascular repair and angiogenesis in the adult due to their rarity. Furthermore, efforts to identify characteristic cell surface markers for ECFCs have not yet been successful.

The controversy regarding the nature of circulating EPCs has resulted in a gradual shift in the field of EPC research and vascular repair. There is a growing realization that resident endothelial cells may be more critical for vascular repair than circulating "EPCs". The supply of de novo endothelial cells as building blocks for vascular growth and repair may require the differentiation of endothelial cells from pluripotent stem cells or the transdifferentiation of fibroblasts into endothelial cells. The current review will highlight recent research in three areas: 1) Contribution of resident endothelial cells to vascular repair (Figure 1A), 2) differentiation and transdifferentiation into EPCs (Figure 1B) and 3) novel paracrine mechanisms by which EPCs may promote angiogenesis and repair (Figure 1C).

\section{Contribution of proliferative resident endothelial cells to vascular repair}

There is a growing body of literature which indicates that there is minimal or no long-term engraftment of circulating EPCs into blood vessels undergoing repair after injury. Instead, proliferative resident ECs or EPCs provide the endothelial cells necessary to replenish the 
injured endothelium (Figure 1A). Recently, Hagensen et al compared the relative contribution of circulating cells and residents cells to vascular repair and concluded that resident arterial ECs primarily contribute to arterial endothelial regeneration [4]. In this study, wire-injured carotid artery segments from wild type mice were transplanted into Tie2GFP mice, in which both mature arterial ECs and circulating EPCs expressed GFP. The study demonstrated that the neo-endothelium was negative for GFP, thus indicating that the regenerative endothelial cells were derived from adjacent ECs contained within the transplanted arterial segment, and not from circulating GFP-positive EPCs. This principle of resident tissue endothelial cells being the primary driver of vascular repair not only applies to endothelial cells in large vessels. Wang et al were able to show that resident liver sinusoidal ECs/EPCs were the main source for providing neo-endothelium during physiologic endothelial turnover of liver sinusoidal ECs in adult rats [5].

It is likely that tissue resident ECs/EPCs are not homogenous and probably represent a hierarchy of endothelial cells with varying degrees of proliferative and regenerative potential, but the specific markers for the most regenerative endothelial cells still remain to be identified. Fang et al [6] isolated a small population of c-kit positive endothelial cells (ckit+Lin-CD31+CD105+ cells) from adult mouse lung that can form highly proliferative colonies. They showed that those cells had long-term self-renewal capacity, a defining functional feature of adult stem and progenitor cells. Thus, Fang et al designated those cells as vascular endothelial stem cells (VESCs), although they offered little evidence for the pluripotency of the cells. They further showed that a single c-kit VESC could generate perfused functional vessels in vivo in mice. On the other hand, c-kit deficient mice had impaired blood vessel formation as evidenced from impaired tumor angiogenesis and retardation of tumor growth in those mice. Whether c-kit(+) endothelial cells are both necessary and sufficient for endothelial repair and whether this principle can be extrapolated to other organs and even human tissues still needs to be determined.

It is still not clear whether activation of quiescent tissue-resident endothelial cells to re-enter cell cycle and regenerate the vasculature always requires a de-differentiation step to a less mature EPC state. It has been reported [7] that ECs from different organs exhibit a unique, vascular-bed specific expression pattern for angiocrine factors (growth factors or cytokines/ chemokines released by the endothelium) and transcription factors that regulate endothelial proliferation, further underscoring that regenerative signals for endothelial cells are not universal but tissue-specific. For example, Ding et al [8] reported that CXCR7-Id1 was a pro-regenerative angiocrine signal in response to liver sinusoidal endothelial cell injury. Interestingly, they found that the relative recruitment of CXCR7-Id1 versus FGFR1-CXCR4 affected the balance between endothelial regeneration and fibrosis in hepatic regeneration. Besides angiocrine factors, transcription factors regulating endothelial proliferation are also key determinants of vascular repair. Our group [9] has shown that a population of lung endothelial cells enter the cell-cycle and may de-differentiate into an immature EPC state after endotoxin induced microvascular injury. Lung endothelial cells fail to proliferate and promote regeneration after vascular injury in mice deficient for the transcription factor FoxM1, thus indicating its important for regeneration of the lung endothelium. 


\section{Differentiation and transdifferentiation into EPCs}

Vascular repair can also be augmented by supplying exogenous proliferative ECs/EPCs which are derived from pluripotent stem cells such as embryonic stem cells (ESCs) or induced pluripotent stem cells (iPSCs). These are especially attractive for therapeutic repair because iPSCs can be derived from any given individual's own fibroblasts (Figure 1B). The generation of iPSCs from fibroblasts was first reported by Takahashi et al in 2006[10]. They showed that adult fibroblasts could be reprogrammed into embryonic stem cell like state by transfecting with four pluripotency transcription factors or "Yamanaka factors":Oct3/4, Sox2, c-Myc, and Klf4. Schenke-Layland et al [11] successfully derived human ECs/EPCs from iPSCs and Yamamoto et al [12] reported that human iPSC derived Flk-1+ EPCs can contribute to re-endothelialization in a mouse femoral artery steel wire injury model. In this study, iPSC derived Flk-1+ EPCs were recruited to the injured site and the cell recruitment was augmented by application of the chemokine Stromal cell-derived factor-1a.

One of the key limitations of deriving EPCs from iPSCs is that it requires 4-8 weeks to generate iPSCs, which then have to be tested for their degree of pluripotency and any genetic or epigenetic abnormalities that may have been generated during the reprogramming process. The iPSCs are subsequently differentiated into EPCs and any cells with residual pluripotency have to be carefully removed since they have the potential for giving rise to teratomas. Thus, there is a growing interest in the direct reprogramming or transdifferentiation of fibroblasts into proliferative ECs/EPCs without inducing pluripotency. Multiple studies have recently been able to successfully reprogram fibroblasts into cells that exhibit an endothelial phenotype. Kurian et al [13] used a short-term expression of pluripotency factors but avoided the induction of full pluripotency and instead induced endothelial differentiation by exposing partially reprogrammed cells to vascular growth factors. This approach generated cells which expressed selected endothelial surface markers and were able to form blood vessels in vitro and in vivo. Using modification of the approach in which cells were transduced with only two instead of four pluripotency transcription factors, Li et al [14] also showed that endothelial-like cells could be generated from human fibroblasts. 8-Br-cAMP and the TGF $\beta$ inhibitor SB431542 were added to increase differentiation efficiency and this also increased the expansion potential of the generated cells. Importantly, in this study, transplantation of the induced ECs showed therapeutic benefits in an experimental peripheral ischemia mouse model: capillary density and blood perfusion were significantly increased in ischemic limbs and immunostaining showed that transplanted human induced ECs engrafted into murine vessels.

Other cell types besides fibroblasts have also been utilized in induction of endothelial cells. Ginsberg et al [15] directly reprogrammed human amniotic cells into endothelial cells by expressing the vascular developmental transcription factors of the ETS family and concomitantly suppressing TGF $\beta$ signaling. Compared with hESC derived ECs, the induced ECs were more proliferative. More importantly, they stably expressed mature EC markers after maintaining the cells in culture for multiple passages, while hESC derived EPCs lost mature EC makers and instead expressed smooth muscle marker smooth muscle a-actin (SMA). In a mouse partial hepatectomy model, the induced ECs were able to engraft and form functional vasculature for up to 3 months. 


\section{Novel paracrine mechanisms by which EPCs may promote angiogenesis and repair}

It is now thought that the primary mode of contribution to vascular repair by circulating $\mathrm{EPCs} / \mathrm{ECs}$ is via the release of paracrine factors. This may explain the therapeutic benefits of transplanted circulating EPCs despite the fact that they do not engraft into the vasculature (Figure 1C). Recent studies have identified novel paracrine mechanisms by which circulating cells may contribute to vascular repair. Baker et al [16] showed that EPC paracrine factors promoted proliferation and angiogenesis of fetal pulmonary artery endothelial cell (PAEC) and alveolar type 2 epithelial cell in vitro. They also showed that the recovery of bleomycin induced bronchopulmonary dysplasia (BPD) in newborn rats benefited from intravenous injection of EPC conditioned medium.

Recently another mode of paracrine action is drawing attention: EC-derived microparticles (EMPs) or microvesicles (MVs). EMPs can induce endothelial regeneration by promoting proliferation of mature endothelial cells [17]. EMPs are small membrane fragments released from activated or apoptotic ECs after injury and contain DNA, RNA or microRNA, which in turn are taken up by target cells where they can activate an angiogenic program [18]. Cantaluppi et al[19] reported that injected EMPs mitigate acute kidney injury in rats by enhancing tubular cell proliferation and decreasing apoptosis. Notably, the EMPs protective effect was reduced by RNase or microRNA depletion in EPCs, indicating that RNA and microRNA in EMPs were important for the vascular repair. Furthermore, Jansen et al [20] showed that injected EMPs accelerated endothelial repair after electric endothelial denudation of carotid artery in mouse. More importantly, by performing TaqMan microRNA array analysis of 384 microRNA, Jansen et al found that microRNA-126 contained in EMPs played critical roles in regulating EC migration, proliferation and regeneration process through inhibiting SPRED1 and promoting Ras/MAPK signaling.

\section{Conclusion}

Recent studies suggest that tissue-resident ECs and EPCs are the key source of endothelial cells for vascular repair. Mechanisms that trigger the regeneration process of resident ECs and EPCs are still under investigation. Since ECs from distinct organs exhibit a significant degree of heterogeneity in terms of their gene expression profile and phenotype, it is likely that the mechanisms underlying endothelial activation and repair are tissue specific. If the regenerative capacity of the resident endothelium is exhausted, supply of exogenous ECs/ EPCs derived from ESCs, iPSCs or converted fibroblasts can help provide the necessary building blocks for vascular repair. The therapeutic potential for resident ECs/EPCs or exogenous EC/EPCs will depend on a better understanding of the tissue-specific mechanisms of endothelial regeneration.

\section{Acknowledgments}

This work was supported by grants from the National Institutes of Health HL090152 (ABM), GM094220 (JR) and HL118068 (ABM and JR). 


\section{References}

Papers of particular interest, published within the annual period of review, have been highlighted as:

- of special interest

•• of outstanding interest

1. Asahara T, Murohara T, Sullivan A, Silver M, van der Zee R, Li T, Witzenbichler B, Schatteman G, Isner JM. Isolation of putative progenitor endothelial cells for angiogenesis. Science. 1997; 275:964-967. [PubMed: 9020076]

2. Rehman J, Li J, Orschell CM, March KL. Peripheral blood "endothelial progenitor cells" are derived from monocyte/macrophages and secrete angiogenic growth factors. Circulation. 2003; 107:11641169. [PubMed: 12615796]

3. Yoder MC, Mead LE, Prater D, Krier TR, Mroueh KN, Li F, Krasich R, Temm CJ, Prchal JT, Ingram DA. Redefining endothelial progenitor cells via clonal analysis and hematopoietic stem/ progenitor cell principals. Blood. 2007; 109:1801-1809. [PubMed: 17053059]

4•. Hagensen MK, Raarup MK, Mortensen MB, Thim T, Nyengaard JR, Falk E, Bentzon JF. Circulating endothelial progenitor cells do not contribute to regeneration of endothelium after murine arterial injury. Cardiovascular Research. 2012; 93:223-231. This study used genetic lineage tracing and a carotid artery transplantation model to demonstrate the contribution of tissue resident ECs but not circulating EPCs to endothelial regeneration. [PubMed: 22012957]

5. Wang L, Wang XD, Xie GH, Wang L, Hill CK, DeLeve LD. Liver sinusoidal endothelial cell progenitor cells promote liver regeneration in rats. Journal of Clinical Investigation. 2012; 122:1567-1573. [PubMed: 22406533]

6. Fang S, Wei J, Pentinmikko N, Leinonen H, Salven P. Generation of functional blood vessels from a single c-kit+ adult vascular endothelial stem cell. PLoS Biol. 2012; 10:e1001407. [PubMed: 23091420]

7•. Nolan DJ, Ginsberg M, Israely E, Palikuqi B, Poulos MG, James D, Ding BS, Schachterle W, Liu Y, Rosenwaks Z, et al. Molecular signatures of tissue-specific microvascular endothelial cell heterogeneity in organ maintenance and regeneration. Dev Cell. 2013; 26:204-219. This is an important study demonstrating the heterogeneity and molecular signatures of ECs obtained from different organs. [PubMed: 23871589]

8. Ding BS, Cao Z, Lis R, Nolan DJ, Guo P, Simons M, Penfold ME, Shido K, Rabbany SY, Rafii S. Divergent angiocrine signals from vascular niche balance liver regeneration and fibrosis. Nature. 2013

9. Zhao YY, Gao XP, Zhao YD, Mirza MK, Frey RS, Kalinichenko VV, Wang IC, Costa RH, Malik AB. Endothelial cell-restricted disruption of FoxM1 impairs endothelial repair following LPSinduced vascular injury. Journal of Clinical Investigation. 2006; 116:2333-2343. [PubMed: 16955137]

10. Takahashi K, Yamanaka S. Induction of pluripotent stem cells from mouse embryonic and adult fibroblast cultures by defined factors. Cell. 2006; 126:663-676. [PubMed: 16904174]

11. Schenke-Layland K, Rhodes KE, Angelis E, Butylkova Y, Heydarkhan-Hagvall S, Gekas C, Zhang R, Goldhaber JI, Mikkola HK, Plath K, MacLellan WR. Reprogrammed mouse fibroblasts differentiate into cells of the cardiovascular and hematopoietic lineages. Stem Cells. 2008; 26:1537-1546. [PubMed: 18450826]

12. Yamamoto T, Shibata R, Ishii M, Kanemura N, Kito T, Suzuki H, Miyake H, Maeda K, Tanigawa $\mathrm{T}$, Ouchi N, Murohara T. Therapeutic reendothelialization by induced pluripotent stem cells after vascular injury--brief report. Arterioscler Thromb Vasc Biol. 2013; 33:2218-2221. [PubMed: 23868941]

13••. Kurian L, Sancho-Martinez I, Nivet E, Aguirre A, Moon K, Pendaries C, Volle-Challier C, Bono F, Herbert JM, Pulecio J, et al. Conversion of human fibroblasts to angioblast-like progenitor cells. Nat Methods. 2013; 10:77-83. This study demonstrated the successful generation of EPCs from fibroblasts without inducing complete pluripotency. [PubMed: 23202434] 
14. Li J, Huang NF, Zou J, Laurent TJ, Lee JC, Okogbaa J, Cooke JP, Ding S. Conversion of human fibroblasts to functional endothelial cells by defined factors. Arterioscler Thromb Vasc Biol. 2013; 33:1366-1375. [PubMed: 23520160]

15. Ginsberg M, James D, Ding BS, Nolan D, Geng F, Butler JM, Schachterle W, Pulijaal VR, Mathew S, Chasen ST, et al. Efficient direct reprogramming of mature amniotic cells into endothelial cells by ETS factors and TGFbeta suppression. Cell. 2012; 151:559-575. [PubMed: 23084400]

16. Baker CD, Seedorf GJ, Wisniewski BL, Black CP, Ryan SL, Balasubramaniam V, Abman SH. Endothelial colony-forming cell conditioned media promote angiogenesis in vitro and prevent pulmonary hypertension in experimental bronchopulmonary dysplasia. American Journal of Physiology-Lung Cellular and Molecular Physiology. 2013; 305:L73-L81. [PubMed: 23666751]

17. Dignat-George F, Boulanger CM. The Many Faces of Endothelial Microparticles. Arteriosclerosis Thrombosis and Vascular Biology. 2011; 31:27-33.

18. Deregibus MC, Cantaluppi V, Calogero R, Lo Iacono M, Tetta C, Biancone L, Bruno S, Bussolati $\mathrm{B}$, Camussi G. Endothelial progenitor cell derived microvesicles activate an angiogenic program in endothelial cells by a horizontal transfer of mRNA. Blood. 2007; 110:2440-2448. [PubMed: 17536014]

19. Cantaluppi V, Gatti S, Medica D, Figliolini F, Bruno S, Deregibus MC, Sordi A, Biancone L, Tetta C, Camussi G. Microvesicles derived from endothelial progenitor cells protect the kidney from ischemia-reperfusion injury by microRNA-dependent reprogramming of resident renal cells. Kidney International. 2012; 82:412-427. [PubMed: 22495296]

20•. Jansen F, Yang X, Hoelscher M, Cattelan A, Schmitz T, Proebsting S, Wenzel D, Vosen S, Franklin BS, Fleischmann BK, et al. Endothelial Microparticle-Mediated Transfer of MicroRNA-126 Promotes Vascular Endothelial Cell Repair via SPRED1 and Is Abrogated in Glucose-Damaged Endothelial Microparticles. Circulation. 2013; 128:2026-2038. This study demonstrated that microRNA-126 contained within microparticles released by EPCs is critical for endothelial repair. [PubMed: 24014835] 


\section{Key points}

- Endothelial progenitor cells (EPCs) that reside within the tissue may be more important for vascular regeneration than circulating EPCs

- Transdifferentiation of fibroblasts and vascular differentiation of pluripotent stem cells provide EPCs necessary for regeneration.

- Circulating EPCs may not directly contribute to the neovasculature but can enhance regeneration by releasing microparticles and exosomes. 


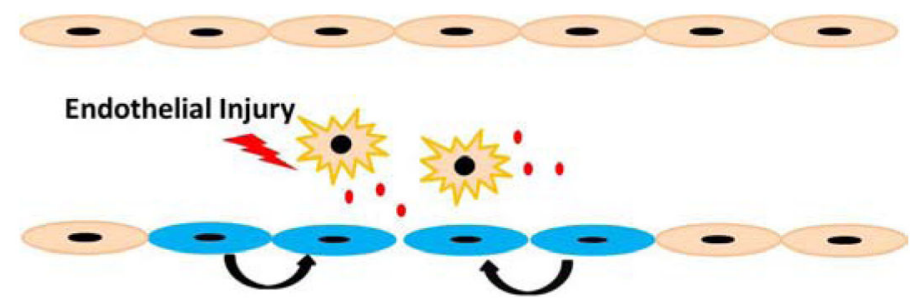

Figure 1A.

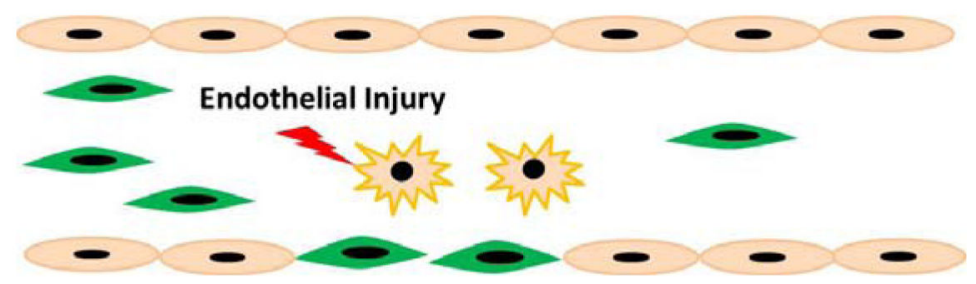

Figure $1 B$.

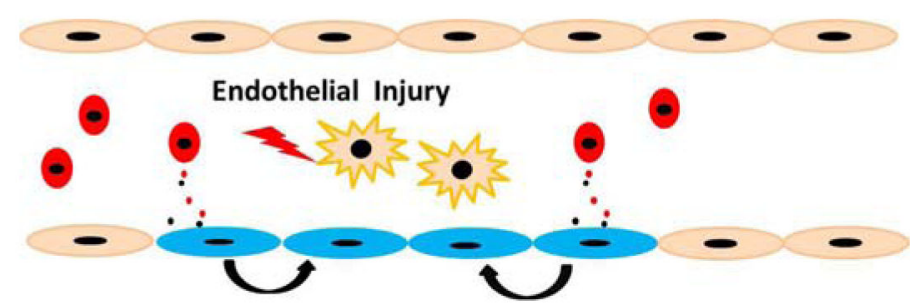

Figure 1C.

Figure 1.

Figure 1A. Vascular repair by endogenous resident ECs. Vascular ECs are injured and undergo apoptosis or necrosis. The cell-cell junctions between injured ECs and resident adjacent ECs are destroyed and intercellular factors are released, which may in turn trigger the proliferation of endogenous resident ECs proliferate to replace injured ECs.

Figure 1B. Vascular repair by exogenous pluripotent stem cells (PSCs) derived ECs/ EPCs. Injected exogenous EPCs derived from ESC/iPSC or directly reprogrammed fibroblasts can integrate into the blood vessel and replenish damaged vascular cells.

Figure 1C. Vascular repair by circulating EPCs. Circulating EPCs can secrete microparticles and paracrine growth factors which activate resident ECs to proliferate and regenerate the injured vasculature

- Resident vascular ECs

* Injured resident ECs 
$\because$ Paracrine factors released from injured ECs

- Activated resident ECs

- Exogenous ECs/EPCs derived from PSCs or directly reprogramed fibroblasts

- Endogenous circulating EPCs

$\therefore$ Microparticles and paracrine factors secreted by circulating EPCs 